\title{
Control Access to a Resource in an E-Learning Platform
}

\author{
Imane Moussaoui ${ }^{1}$, Hafida Essaoudi ${ }^{2}$, Mohamed El Ouaazizi ${ }^{1}$, Abdelaziz \\ Hallaoui ${ }^{1}$, Aziza El Ouaazizi, Abdelouahed Essahlaoui ${ }^{1, *}$ \\ ${ }^{1}$ Engineering Sciences Laboratory, Multidisciplinary Faculty of Taza, Sidi Mohamed Ben Abdellah University, \\ B.P. 1223, Taza gare, Taza, Morocco \\ ${ }^{2}$ Faculty of Sciences, Moulay Ismail University, B.P.11201, Zitoune, Meknes, Morocco.
}

\begin{abstract}
With the huge technological progress, Technology fever has invaded all social and economic fields and one of these fields is the educational one. The e-learning systems which are based on the principle of the online courses, these systems are the most important aspects of the involvement of technology in education. However, when we are talking about any computer system using any kind of networks we are supposed to treat the security issue of this system. Although the security issue is important, it hadn't got much attention in the development of an e-learning platform. In this article we're going to expose some vulnerability that we can find in the connecting bridge between the profiles of learners and other resources in the e-learning platform.
\end{abstract}

Keywords: Security, e-learning, vulnerability, Profile of learners, control access.

*Corresponding author:abdelouahed.essahlaoui@usmba.ac.ma

\section{Introduction}

The field of education has progressed in a very remarkable way during the last years. One of the major reasons of this development is the involvement of Human Learning System (HLS) in different ways in the education system in all over the world. The HLS has an immeasurable importance because of its flexibility and portability, also because of its great contribution in improving the learning domain [1]. The HLS accord to its users the possibility to learn by choosing their own way and strategy in learning. Therefore these online platforms help the learners to find their own learning style [2,3]. Although the diversity of the HLS platforms is on a rapid growth but all these platforms are online, as they growth as they are more and more exposed to a lot security threats. That is why the security techniques [4-6] must have a preponderant importance in this kind of platforms.

The presented work in this article is associated to the project "the formalization of a general security policy for all types of e-learning platforms" which aims to develop a global study of all the security aspects and its role in the improvement of the e-learning field. Our research focuses on all types of software security (data security, network security...) and their involvement in the development of a less vulnerable platform. In this paper we will expose some threats that we can find in an e-learning platform and we will focus on how to assign the right of access to a resource for a learner model.

For this we ask several questions:

- How can we assign the right of access to a learner?

- How can we allow or deny a learner to access to an existing resource automatically?

- Is these access rights can be assigned automatically?

In this paper we will answer these questions and we will apply our suggestion to a model of profile learner inspired by another one already existing.

\section{Studying The Existing}

Before discussing our point we will introduce this paper by a brief explanation of the concepts which we will use.

\section{a. Difference between HLS \& Web-based Training and e-learning :}

Before starting our discussion, we have to clarify the ambiguity between three different terms that are usually used for the same thing. For this reason we give to each term its own definition:

- Web-based training: this training fits into the framework of distance learning. It is a training program accessible via a web browser or internet or even intranet, the program should essentially use the web.

- E-learning: it consists on using internet and computer resources in order to make learners able to acquire knowledge with remote access.

- HLS: it's a computer environment designed to promote human learning, it's generally used to personalize learning. It's also used in face interaction situations or remotely. There are a lot of types of these environments for example: Hypermedia, platforms of distance learning...

In our article we will opt for the HLS (Human learning systems) as the trial system. 


\section{b. Profile learner :}

In an e-learning system, one of the most powerful components is the learner profile. It can be defined as a data structure including the majority of information about the learner. It can have a lot of forms, it can be a normal document (paper for example) or it can be a set of information related to the learner stored in a structure in a given system. However this information about the learner is not only personal, it's also a set of other ones like his assessment, strengths, skills, interests, and behaviors. Therefore this profile can be considered as a dynamic one, because of the mutations of all these components of the profile gradually as his period of learning. Other researchers also explained in the profile, the metacognitive information of the learner [7-9].

\section{c. Right access in Security:}

Every developer of a computing environment should understand the importance of the security information, and should take the essential measures in order to prevent its system from the vulnerabilities. One of the fundamental aspects of security information is the control of rights of access to every resource of the system. This aspect is one of the major methods that allow us to protect our system against attacks by third parties and attackers. The idea of our article will be based on the control of right access to a resource in the learning environment.

\section{Related Researches}

There are diverse studies which have been done on the subject of security and its role on improving the exploitation of E-learning platforms. Ciobanu Costinela et al. [4] treated the HLS vulnerabilities, they took MOODLE as a case study and they studied the effect of the SQL injection attack on this platform. I. Bandara et al. [10] did a research about the cyber security concerns in E-learning education and they proposed a security management model for E-learning systems. But all these studies were dedicated to the e-learning platforms and few of them discussed the security in HLS platforms. Jeghal et al. [11] did a research about the politic of security in human learning systems and they proposed a policy which insures the safety of profile learners. This study was based on a previously proposed model by Jeghal et al. [12].

\section{Methodology And Discussion:}

Our Contribution will be based on the previously proposed model by Jeghal et al[11] in order to improve this model and to highlight the control access of the learners to some resources in the platform. In other hand we will choose the course as an example of a resource in an e-learning system. Before starting our discussion we would like to give an example of the way to access to a course via an e-learning platform for example: MOODLE.

\section{A case study of the rights of access to a course on moodle platform: \\ a. Moodle Platform:}

Moodle (Modular Object-Oriented Dynamic Learning Environment) is a free open-source LMS (learning management system) or e-Learning platform. It has over 60 million users in all over the world and was developed by Martin Dougiamas.

\section{b. Options to access to a course on Moodle:}

In order to control the access to a course on Moodle Platform there are 3 settings which can be set by the administrator, the author or the tutor:

- Registration Key

- Guest access: Anonymous users' access not allowed or allowed with the key or allowed without key.

- Availability: If this course is open to students or not.

For example the tutor doesn't need to register one by one his students to his classes. He simply creates a registration key and spread it to his students. Then the student will access to the course by providing this key.

For the anonymous users there are 2 cases:

- The access to the course might be restricted by the registration key, that is to say that a person who has no account on Moodle can access to the course provided that that person knows the registration key, but unlike the identified user, the anonymous one has to provide the key each time he wants to access to the course.

- The access to the course is open to all the visitors of the platform.

\section{c. Limits of the access rights provided by the HLS platforms}

The access parameters to a course are putted by the administrator of the platform or by the tutor or the author of the course himself. To wrap up this case study shows us that the rights of access to some resources are set by the actors in the HLS platforms but it cannot be guaranteed automatically. That's why we will present a solution that will allow a learner to access to a course according to the relation between the configuration of its 
profile and the course configuration. For this we will propose a model of a profile of a learner and a model of a course.

\section{Solution:}

\section{a. Architecture of an e-learning platform:}

The figure above describes a simplified architecture of an e-learning system. Our paper will focus on the three colored axes in the figure "the learner" which is represented in the system by its profile, "the educational resources" and the connection between them.

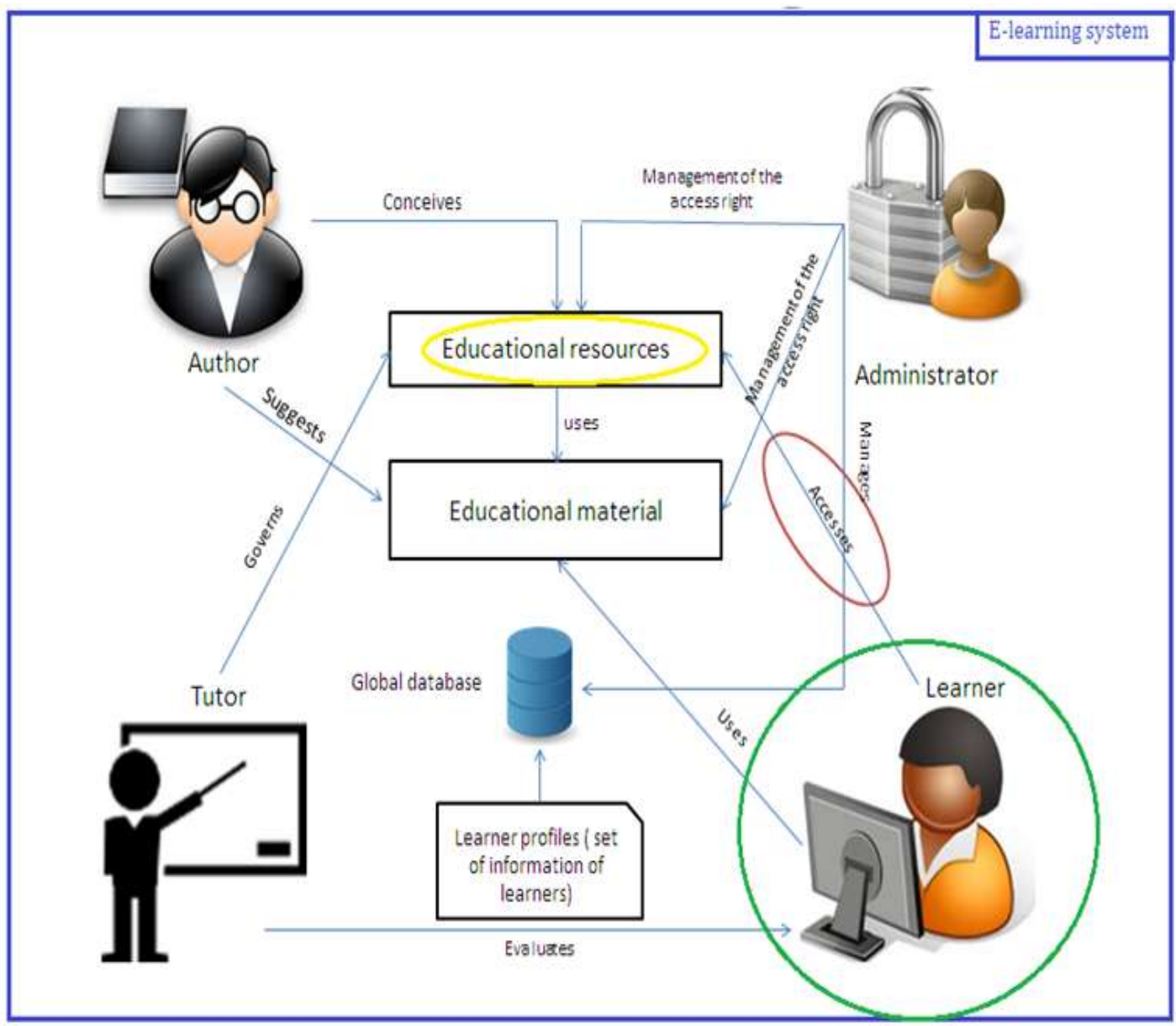

Figure 1: Simplified architecture of an e-learning platform

As we can deduce all the rights access are managed by the administrator. The idea is to make use of the information related to the learner which is stored into the profile (preferences, assessment...) in order to authorize or deny the access to a resource in the platform. In this way the security measures will be reinforced specially the access rights to a resource. In our paper we will use the course as a resource and the evaluation of a learner.

\section{b. Model Proposed:}

It has already been proposed to represent a learner profile [12] in the system by using XML. This Structure of profile proposed contains all the necessary information about the learner, its behavior in the HLS, its skills and knowledge. The choice of XML files to store the profile is based on the fact that the XML points out simplicity, generality and usability.Inspired by this model specially the fact of structuring the model of profile following the form proposed : <Category, Subcategory, Action>. We will create a profile in which the access parameters are set automatically. For this we will represent only a part of our new model of profile in the following figure: 


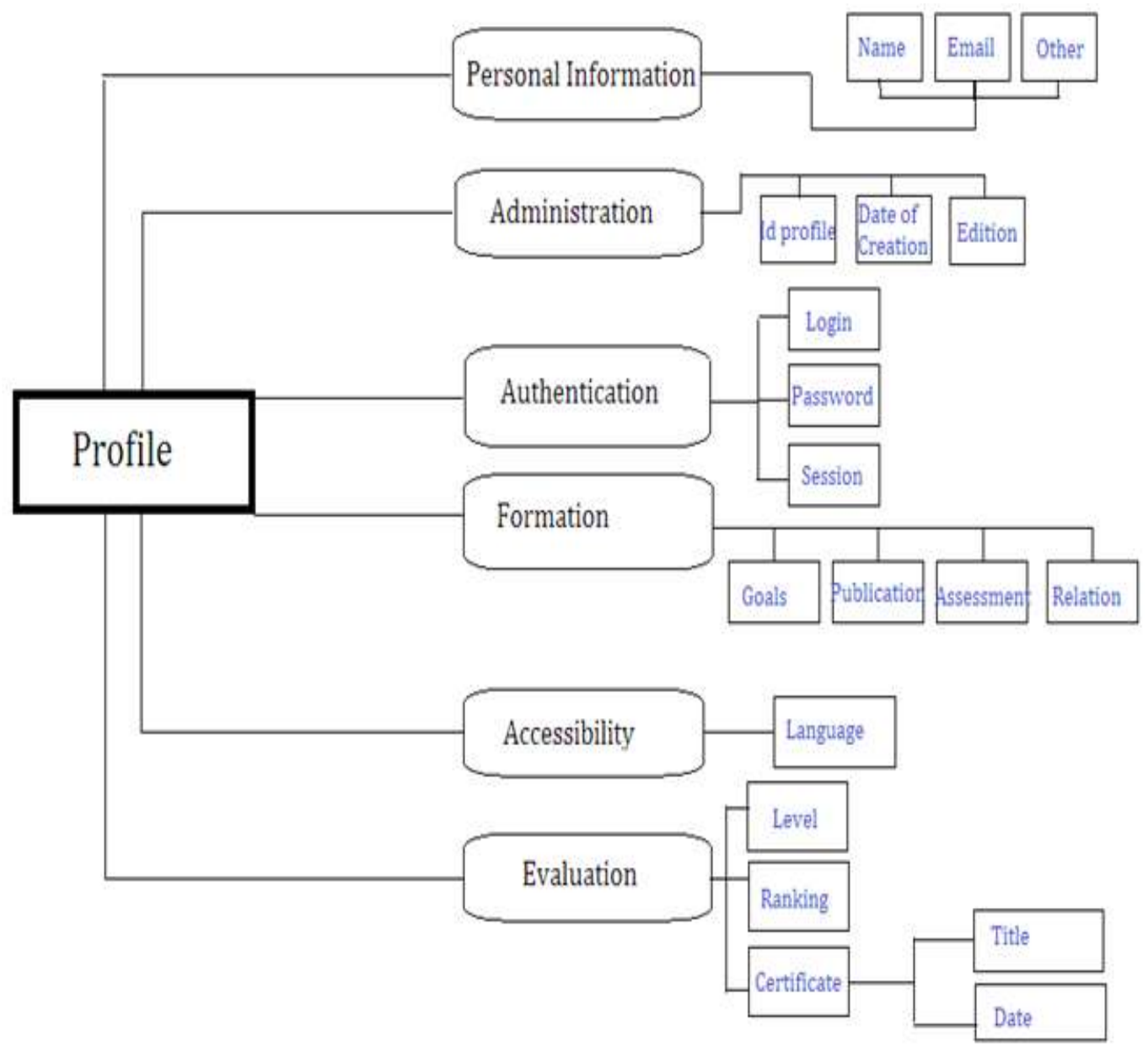

Figure 2: A part of our learner profile

\section{c. Proposed solution to access to a course:}

In general the right to access to a course is set by one of the 3 following users of the HLS: the administrator, the tutor or the author. We are going to set this right automatically by generating some xml files to either the course model of the model of the profile learner.

\section{- The course:}

When one of the 3 allowed users tries to add a course in the platform for example for Moodle (Fig 2). A field should be added to the settings of the course which will be named "Level assigned". This field will be in the form of a combo box enumerated from 1 to 5 . The creator of the course should put a value to its course referring to its difficulty. At the same time the system will automatically generate a file which will be named "Information file" (Fig 3). This file will contain some information about the course and the level of the course will be stored in this file. In the Fig 3 we will give an example.

The creator of the course will have to give a quick quiz (multiple choices), we chose the quiz as a way of evaluation because of its simplicity. In the beginning of a session of training if a session contains a lot of courses. This quiz will allow us to evaluate the learner technically and the result of the quiz will allow to the system to give a level to this learner automatically which the value will be stored in a file (next section). If the course doesn't belong to any session then the quiz must appear when the learner attempt to open the course.

But our solution is more beneficial in the first case when a course belongs to a chain of courses which are linked and the learner have already had the necessary skills to ignore some of them but the right to access to more advanced courses is guaranteed only with the permission of the author or after passing the assessment of another course. Our method will allow this right to the learner automatically. 


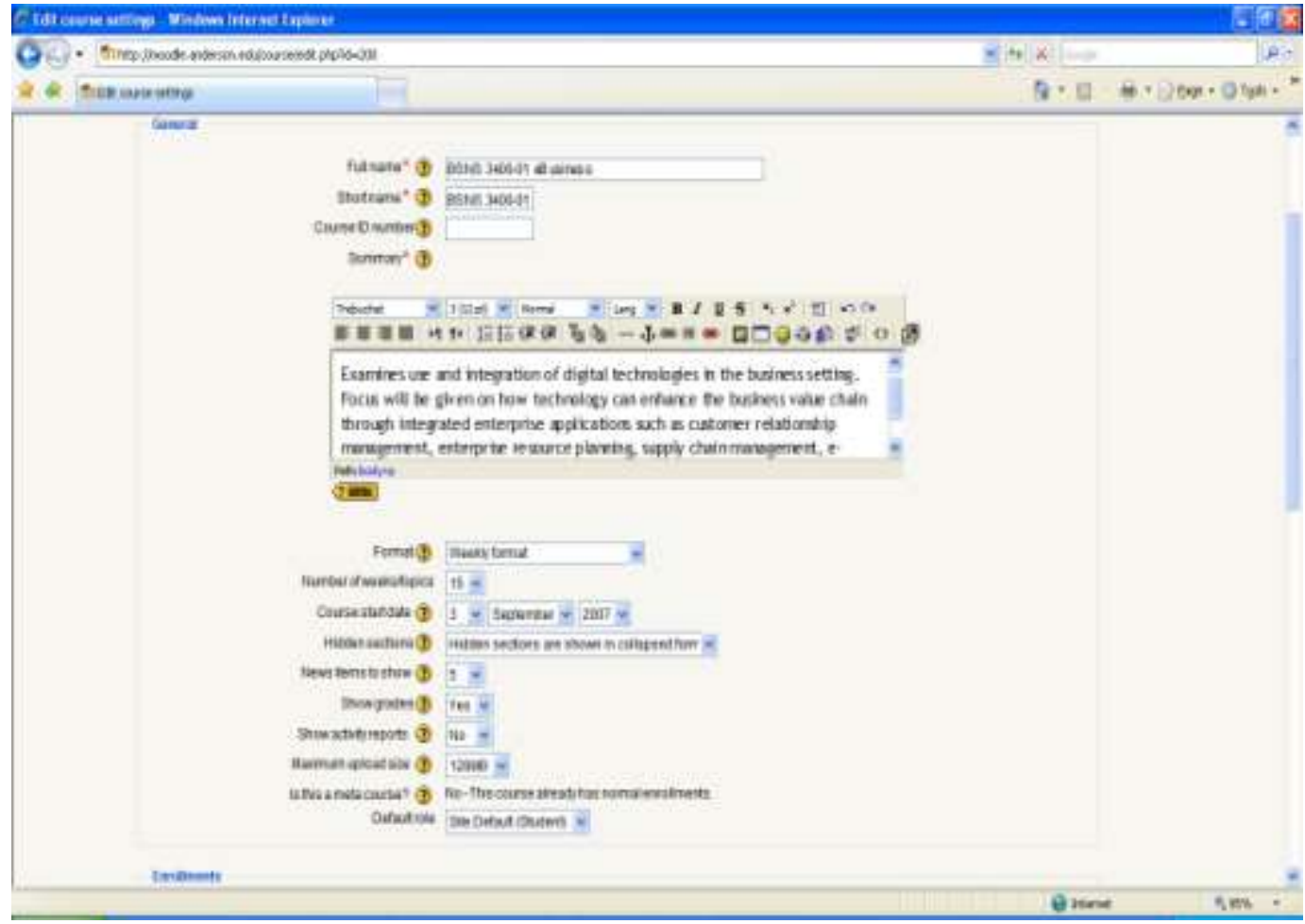

Figure 3: Course parameters window in Moodle

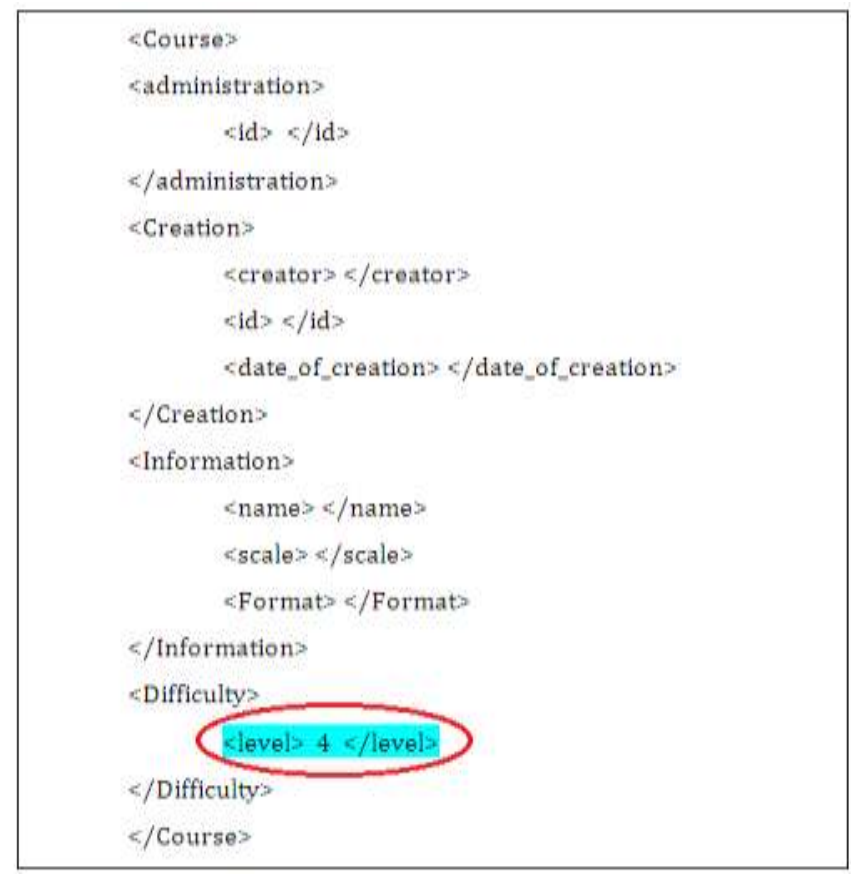

Figure 4: Course structure

\section{- The Model of the learner:}

In the previous researches [13], it was proposed that when the administrator of the system starts creating a profile two files will be generated automatically (Fig 4). Our solution will focus on the data file, we will divide this file into two others one named "Personal data file" and the second will be named "Tracking data file" (Fig 5). All the personal information's learner will be stored in the first one; the second one will contain the ranking, the level, and the evolution of the learner. Our contribution consist that with every session of training a tracking file (Fig 6) will be generated automatically containing some variable values which depends on the evolution of the learner in this session. 
In our case the learner have to pass a quiz in his first connection to the session. When the result of the quiz is set the system will assign automatically a level to our learner. This level stored in our tracking file and the level stored before in the information file of the course have to match if the learner wants to access to the course. Also the level of the learner will allow him to access to any course which the level is inferior to the level of the learner.
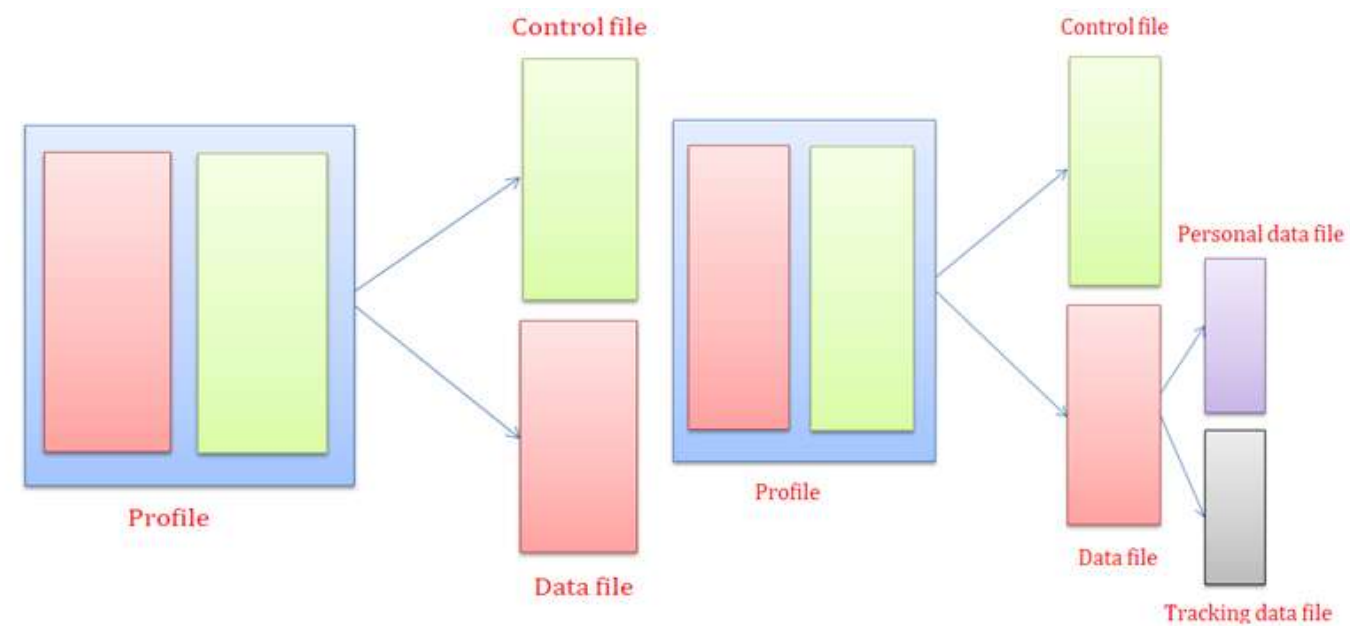

Figure 5: Generated $\mathrm{xml}$ files from the profile

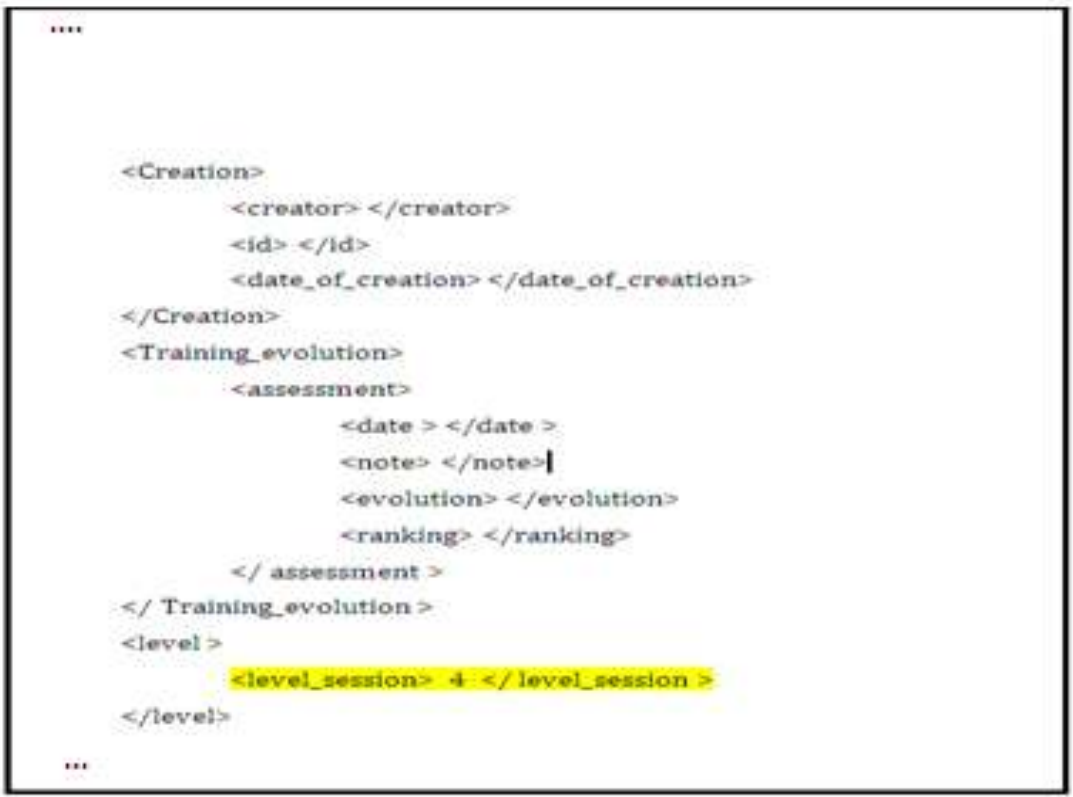

Figure 6: Structure of tracking data file in XML

To implement the method proposed, the system must be provided by a generator of file which will be able to generate the different files needed automatically at the same time of the creation of either a course or a profile.

\section{d. Discussion:}

In the most cases of the HLS, the right to access to a course is set by the either the administrator, or the tutor or the author. Our contribution was about creating a link between the rights to access to a course as example of a resource and the profile of a learner automatically. This method proposed can be improved by using another method of evaluation; also we can use the preferences of the learner to give him the access rights to a resource. Our idea can also guarantee the fact that a resource cannot be reached by everyone. It restricts the chances that someone accesses to a resource if he lacks the skills needed to use it; this is what reinforces the security aspects in the overall system. 


\section{Conclusion}

The e-learning is becoming increasingly important because of the huge mutation of the world of technology. Based on the data processing, the e-learning is a system which needs to be improved. Our paper was about facilitating the way to access to a resource in an e-learning system by using one of its components which is the learner profile. As a perspective we can improve our method by using other information of the learner other than the evaluation in order to facilitate the access to all the resources of the system.

\section{References}

[1]. S. Zhao, Q. Cao, J. Chen, Y. Zhang, J. Tang, Z. Duan, A multi-ATL method for transfer learning across multiple domains with arbitrarily different distribution, Knowledge-Based Systems, Knowledge-Based Systems, Vol. 94 (2016), pp. 60-69.

[2]. A. Ezzat Labib, José H. Canós, M. Carmen Penadés, On the way to learning style models integration: a Learner's Characteristics Ontology, Computers in Human Behavior, Vol. 73 (2017), pp. 433-445.

[3]. J. Bernard, T.-W. Chang, E. Popescu, S. Graf, Learning style Identifier: Improving the precision of learning style identification through computational intelligence algorithms, Expert Systems with Applications, Vol. 75 (2017), pp. 94-108.

[4]. Ciobanu (Defta) Costinela-Luminiţa, Ciobanu (Iacob) Nicoleta-Magdalena,E-learning Security Vulnerabilities, Procedia - Social and Behavioral Sciences, Vol. 46 (2012), pp. 2297-2301.

[5]. Defta Costinela Luminita, Information security in E-learning Platforms, Procedia - Social and Behavioral Sciences, Vol. 15 (2011), pp. 2689-2693

[6]. J. Miguel, S. Caballé, F. Xhafa, Intelligent Data Analysis for e-Learning, (2017), pp. 7-23.

[7]. S. Kostromina, D. Gnedykh,Students' Psychological Characteristics as Factor of Effective Acquisition of Visual Information in Elearning, Procedia - Social and Behavioral Sciences, Vol. 217( 2016), pp. 34-41.

[8]. E. Norman, B. Furnes, The relationship between metacognitive experiences and learning: Is there a difference between digital and non-digital study media, Computers in Human Behavior, Vol. 54 (2016), pp. 301-309.

[9]. B.- J. McGarry, K. Theobald, P.- A. Lewis, F. Coyer,Flexible learning design in curriculum delivery promotes student engagement and develops metacognitive learners: An integrated review, Nurse Education Today, Vol. 35 (2015), pp 966-973.

[10]. I. Bandara, F. Ioras, K. Maher,Cyber security concerns in e-learning education, International Conference of Education, International Conference of Education, Research and Innovation Proceeding, ISBN: 978-84-617-2484-0 (2014), pp. 728-734.

[11]. A. Jeghal, L. Oughdir, H. Tairi,Politic of security, privacy and transparency in human learning systems, Education and Information Technologies, Vol. 21, Issue 3 (2016), pp 521-530.

[12]. A. Jeghal, L. Oughdir, H. Tairi, A. Elaffar,A model of the reuse of the profiles of learners, Journal of Theoretical \& Applied Information Technology, Vol. 57, Issue 2 (2013), pp. 288-293.

[13]. A. Korchi, N. Elamrani Elidrissi, A. Jeghal, L. Oughdir,A modeling learner approach in a computing environment for human learning based on ontology, International Journal of Computer Engineering \& Technology, Vol. 6, Issue 9 (2015), 21-31. 